\title{
Design of a Three-Dimensional Face Recognition System
}

\author{
Wang Xuechun ${ }^{*}$ and Wang Zhaoping
}

School of Information Engineering, Huanghe Science and Technology College, Zhengzhou, Henan, 450006, P.R. China

\begin{abstract}
With the development of computer vision and computer graphics, face recognition technology has gradually developed from 2D to 3D. Using in-depth information of faces, 3D face recognition solves and overcomes the environment and changes in the expression that 2D face recognition encounters. In this paper, the principles of laser triangulation measurement applied in 3D detection and modeling of faces are studied. A 3D face recognition system is designed on this basis. It not only has universal configuration and simple algorithm as well as other characteristics, but also has certain practicality.
\end{abstract}

Keywords: Face recognition, Computer vision, 3D modeling, Principles of triangulation measurement.

\section{INTRODUCTION}

Face recognition based on computer vision technology is widely used in human-computer interaction, identification, virtual reality and other fields. Compared with fingerprints, iris, gene and other biometric identification technology, it is more active, direct, friendly, and subtle and so on [1], therefore has become a very active research topic in the field of computer mode recognition. At the same time, it not only involves a number of research directions of computer vision, digital image processing, pattern recognition and computer graphics, but also makes the computer face recognition technology become a challenging task [2].

At present, methods and theories of 2D face recognition technology are relatively mature, which have made certain achievements [3, 4]. However, based on the principles, 2D face recognition technology will inevitably be affected by light, background, expression, makeup, dressing and other factors. They have brought many restrictions for recognition, thus difficult to establish a robust two-dimensional face recognition system. To solve these problems, many scholars agree that the future for face recognition technology lies in $3 \mathrm{D}$ recognition [5]. The adoption of face recognition technology can better present the in-depth information, topological structure and other facial characteristics. This paper applies principles of laser triangulation and concept of structural light for detection and obtaining of 3D face data, so as to establish data model. Visual Studio from Microsoft is applied as the platform for system development, using graphic image processing functions, combined with OpenGL professional 3D model management engine for analysis and processing of data model, it can implement a 3D face recognition system.

*Address correspondence to this author at the School of Information Engineering, Huanghe Science and Technology College, Zhengzhou, Henan, 450006, P.R. China; Tel: +86 13303820802; E-mail:38856735@qq.com

\section{PRINCIPLES OF FACE DATA COLLECTION}

Face data collection is both the important basis for effective recognition of $3 \mathrm{D}$ facial model and the major research direction. Currently, there are three most common 3D data acquisition methods: first of all, measure facial data through digitizer--facial measurements are obtained directly from digitizer, or structured light sensor, or tomography, and 3D model will be obtained after the measured data is digitized [6]; second, based on general model method, general facial model is generated artificially, and faces are structured through B-spline and other methods, then surface rendering texture information is filled [7-9]; third, 3D facial model is constructed on the basis of a set of two-dimensional images $[10,11]$. Applying the idea of structured light, the paper establishes a platform able to obtain facial 3D data with a laser lighting source and an ordinary camera, and completes a data acquisition system through triangulation principle.

As shown in Fig. (1), the baseline distance between the laser source of data acquisition system and camera as well as the target position that laser is pointing form a vector triangle. The purpose of laser triangulation measurement is to find the target point of space coordinates at a known reference frame (i.e. point $\mathrm{P}$ in Fig. (1). In Fig. (1), L represents the starting point of laser beam to P; A represents the vector between the starting point of laser beam and optical center of the camera lens; $\mathrm{C}$ represents the vector from the optical center of the camera lens to the point $P$.

The geometry relationship is as shown in the following vector equation:

$\vec{L}=\vec{A}+\vec{C}_{\mathrm{xy}}$

In Equation (1), direction cosine of vector $\mathrm{A}$ and vector $\mathrm{C}_{\mathrm{xy}}$ can be obtained through the camera calibration.

If represented by spherical coordinates, Equipment (1) shall be represented as Equations (2) (4), in which $\varphi$ 


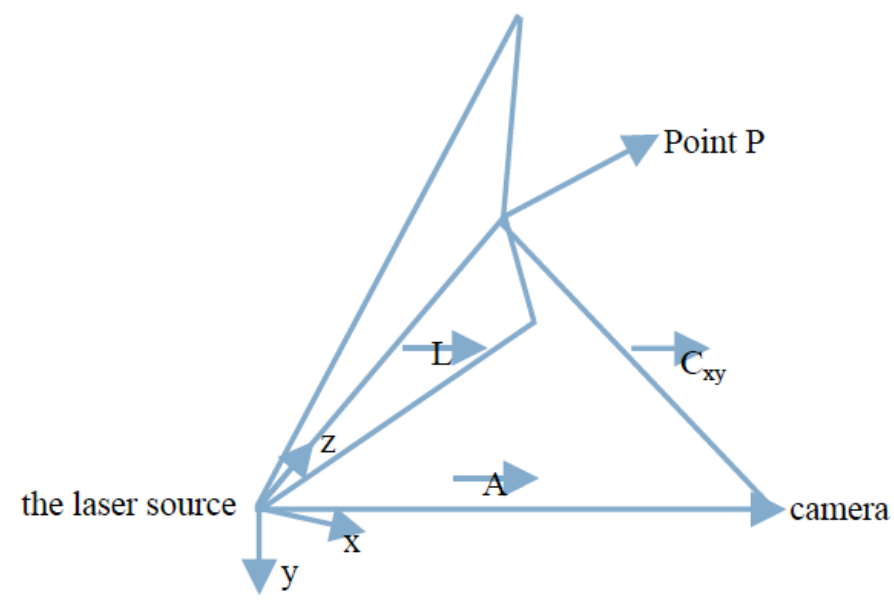

Fig. (1). Triangulation measurement principle diagram.

represents the angle between vector $\mathrm{L}$ and plane $\mathrm{X}-\mathrm{Y}, \theta$ represents the angle of the laser beam between the plane and the $\mathrm{X}$ axis.

$$
\begin{aligned}
& |L| \cos \phi \cos \theta=A_{\mathrm{x}}+C \mathrm{xy}_{\mathrm{x}} \\
& |L| \cos \phi \sin \theta=A_{\mathrm{y}}+C \mathrm{xy}_{\mathrm{y}} \\
& |L| \sin \phi=A_{\mathrm{z}}+C \mathrm{xy}_{\mathrm{z}}
\end{aligned}
$$

For this nonlinear system, it will take very high compute costs to solve these equations. However, $Z$ axis is placed along the laser beam as reference system $(\varphi=0)$, the system can be simplified. The equation of new system is shown in the following Equations (5) (7):

$$
\begin{aligned}
& L_{\mathrm{x}}=|L| \cos \phi=A_{\mathrm{x}}+C \mathrm{xy}_{\mathrm{x}} \\
& 0=A_{\mathrm{y}}+C \mathrm{xy}_{\mathrm{y}} \\
& L_{\mathrm{z}}=|L| \sin \phi=A_{\mathrm{z}}+C \mathrm{xy}_{\mathrm{z}}
\end{aligned}
$$

Analysis of Equations (5) (7) is shown as the following Equations (8) (10):

$L_{\mathrm{x}}=|L| \cos \phi=A_{\mathrm{x}}+|C \mathrm{xy}| \alpha$

$|C x y| \gamma=-\frac{A \gamma}{\beta}$

$L_{\mathrm{z}}=|L| \sin \phi=A_{\mathrm{z}}+C \mathrm{xy}_{\mathrm{z}}$

Wherein $\alpha, \beta, \gamma$ are the direction cosine of vector $\mathrm{C}_{\mathrm{xy}}$ of laser reference system. These parameters can be found combined with the calibration of the camera, thus the solution for this problem can be completed with iterative algorithm.

\section{SOLUTIONS OF FACE RECOGNITION SYSTEM}

The system uses Microsoft Visual Studio as a development platform. With the powerful graphic and image pro- cessing function, OpenGL professional 3D processing engine is applied, coupled with the management functions for heterogeneous data on the carrying Oracle database to realize the face recognition system. The acquisition of facial 3D data applies the $3 \mathrm{D}$ data modeling approach based on the above grating structure. The use case diagram of entire system is shown in Fig. (2).

Management platform conducts the acquisition and preprocessing of facial 3D data with the 3D feature point selection, storage and other features. Using 3D model management system developed by Visual $\mathrm{C}++$ and OpenGL, the original facial $3 \mathrm{D}$ data is obtained through structural light shooting camera. Since the obtained coordination of $3 \mathrm{D}$ data is not uniform, in order to facilitate the calculation, unified coordination is required, so as to smooth the original model through platform. According to the requirements for resolution, surface data of 3D face is generated.

Through analysis of the video stream, the presence of a human face will be automatically detected and split from the image. After splitting the face, calibration of facial feature points will be automatically done. According to the organ composition of head, characteristics of appearance and for the modeling convenience, the head feature designs are classified as structural features and styling features. Structural features include the eyes, nose, mouth and ears; the corresponding styling features are head height, head width, eye width, eye height, eye spacing, nose width, nose height, nose length, mouth width, width of upper and lower lips, ear length, ear width, ear thickness and so on. After the feature points obtained, according to the positions of main feature points, shooting angles are calculated. Since the facial feature points are defined by significant visual characteristics, like the nose tip in the front image usually is the brightest point, but in the side image it is the leftmost point of the entire face. Therefore, it is possible to use image analysis methods to automatically determine their positions. For some specific areas, such as the lips, eyes, etc., by constructing a two-dimensional deformation model, combining with the feature point extraction and model transformation, the accuracy for extraction can be improved and possible errors that noises may bring can be eliminated. 


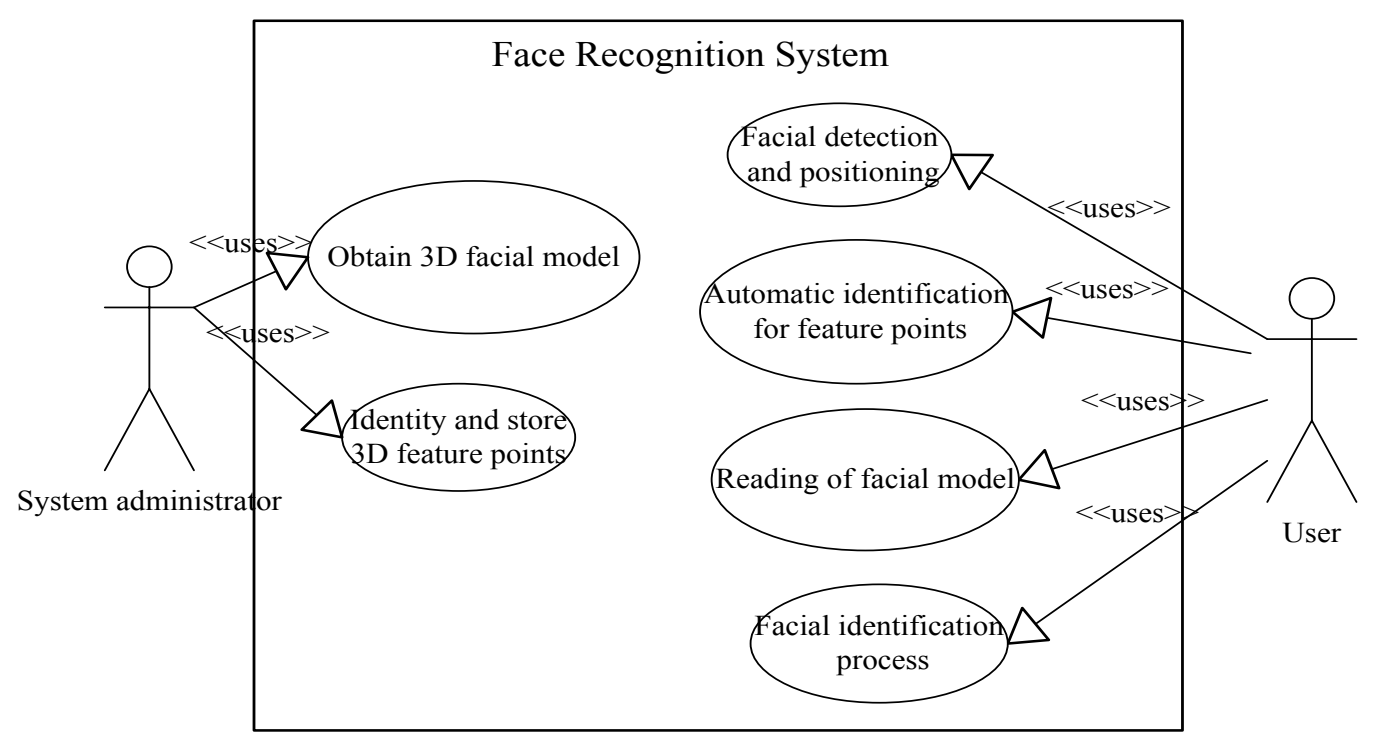

Fig. (2). Use case diagram of the system.

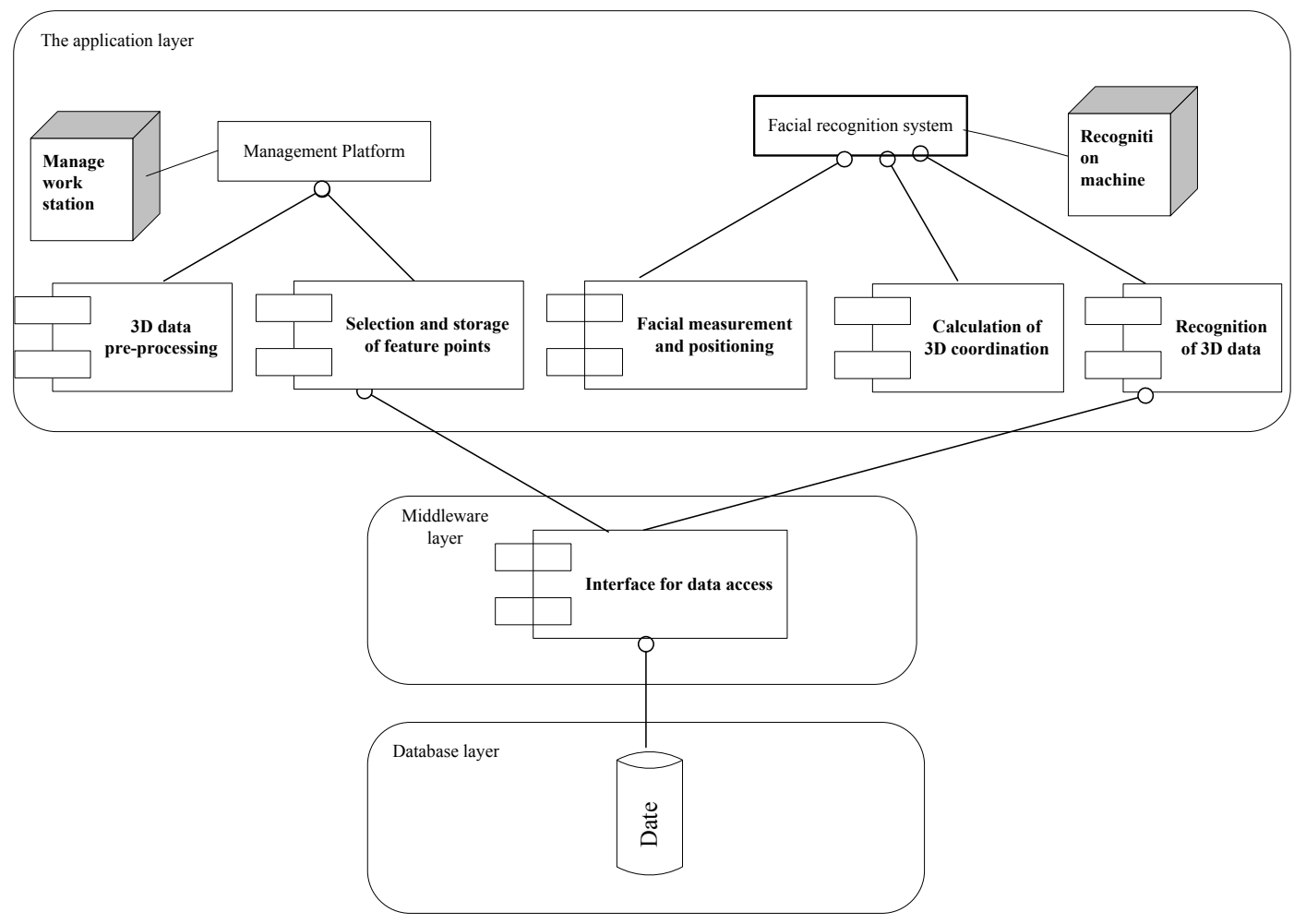

Fig. (3). Structural diagram of systematic structure.

Then read the $3 \mathrm{D}$ data and use OpenGL to construct a three-dimensional model of the human face and compare with the model of changes in shooting angle. While drawing human head model, triangle drawing is selected. Definition for points, lines and polygons in OpenGL are defined by the definition of vertex coordinates, and all the geometric objects are finally drawn in accordance with the definition order of vertex. The generalized facial mesh model we apply is a $3 \mathrm{D}$ polygon mesh model drawn by OpenGL. We select the point data consisting of all features and facial data. Since the coordinates of the vertex and vertex of the triangle surface are known, we can link these points and faces to constitute the mesh model of human head. If the plane projection of $3 \mathrm{D}$ model and rotation angle of human face in $2 \mathrm{D}$ photo are the same, recognition can be achieved by multi-feature-vector generation, multi-classifier discrimination as well as threshold settings.

The underlying database applies Oracle database system. With its good heterogeneous storage and processing capabilities for big data, the unified storage of 3D spatial information and attribute information as well as retrieval of mas- 
sive data queries required for the system can be achieved. 3D face data stored in the database shall contain three parts of information: vertex information, texture information, grid information. (1) Vertex information: the vertex information consists of dense sampling points, and the vertex information of 3D facial model is constituted by these sampling points; (2) Texture information: Texture information describes the corresponding values for each vertex. (3) Grid information: Grid information describes the relationship among vertices. Data in the database uses triangular mesh to describe the links among vertices.

Facial database reading of automatic identification system in the application layer is completed through the middleware, which conducts uniform information reading, maintaining and operating work. Eventually a three-tier $\mathrm{C} / \mathrm{S}$ model application system based on the middleware of 3D database is formed. The overall system design diagram is shown in Fig. (3).

\section{CONCLUSION}

The system solves the problem of multi-pose in face recognition through changes in in-depth information of $3 \mathrm{D}$ projection. Using invariance principles of geometric topology, impact of most facial expression on recognition can be excluded, so as to solve the effect of light intensity in shooting environment during face recognition process and achieve the ideal face recognition results; standard facial 3D data are stored in the database, which have greatly improved the speed of recognition. Therefore, the system has a high value for application.

\section{CONFLICT OF INTEREST}

The authors confirm that this article content has no conflict of interest.

\section{ACKNOWLEDGEMENTS}

This work was financially supported by Zhengzhou Key Laboratory of Embedded System Application Technology, China (Grant No. 121PYFZX177) and Zhengzhou Municipal Natural Science Research Project, China (Grant No.20141372).

\section{REFERENCES}

[1] Z.M. Cai, and J.W. Yan, "Face recognition: from 2D to 3D", Computer Engineering and Applications, vol. 47, pp. 155-159, 2011.

[2] Z.Z. Hu, Estimation of Face Rotation and 3D Face Recognition Based on Depth Data, Hefei University of Technology, Hefei, ON, China, 2011.

[3] P. Chellappa, C.L. Wilson, and S. Sirohey, "Human and machine recognition of faces: a survey", In: Proceedings of the IEEE, vol. 83, pp. 705-740, 1995.

[4] W.Y. Zhao, R. Chellappa, P. J. Philips, and A. Rosenfeld, "Face recognition: A literature survey", ACM Computing Survey, vol. 35, pp. 399-458, 2003.

[5] Z.S. Wu, Research on 3D Facial Surface Construction and Feature Extraction Based on B-splines, Xiamen University, Xiamen, ON, China, 2008.

[6] Y.G. Yang, Analysis and Implementation of Three Dimensional Person Face Recogllition System, Dalian University of Technology, Dalian, ON, China, 2006.

[7] M.D. Cordea, E.M. Petriu, and D.C. Petriu, "Three-dimensional head tracking and facial expression recovery using an anthropometric muscle-based active appearance model", IEEE Transactions on Instrumentation and Measurement, vol. 57, pp. 1578-1588, 2008.

[8] K. Yano, and K. Harada, "A facial expression parameterization by elastic surface model”, International Journal of Computer Games Technology, vol. 2009, Article ID. 397938, 2009.

[9] P. Paysan, R. Knothe, B. Amberg, S. Romdhani, and T. Vetters, "A 3D face model for pose and illumination invariant face recognition", In: Proceedings of the $6^{\text {th }}$ IEEE International Conference on Advanced Video and Signal Based Surveillance, Genoa, Italy, 2009, pp. 2-4.

[10] B.X. Shi, Y. Matsushita, Y.C. Wei, C. Xu, and P. Tan, "Selfcalibrating photometric stereo", In: Proceedings of the $23^{\text {rd }}$ IEEE Conference on Computer Vision and Pattern Recognition, San Francisco, 2010, pp. 13-18.

[11] R. Basri, D. W. Jacobs, and I. Kemelmacher, "Photometric stereo with general, unknown lighting", International Journal of Computer Vision, vol. 72, pp. 239-257, 2007. 